\title{
Autoimmune pulmonary alveolar proteinosis with progressive fibrosis refractory to treatment with whole lung lavage, inhaled GM-CSF and rituximab
}

Cheryl R. Laratta ${ }^{1}$, Kerri A. Johannson ${ }^{2}$, Margaret M. Kelly ${ }^{3}$, Matthew J. van Olm², Andrew G. Lee ${ }^{4}$, Charlene D. Fell $^{* 2}$

${ }^{1}$ Division of Respirology, University of Alberta, Canada

${ }^{2}$ Division of Respirology, University of Calgary, Canada

${ }^{3}$ Department of Pathology, University of Calgary, Canada

${ }^{4}$ Department of Radiology, University of Calgary, Canada

Received: November 29, 2016

Accepted: January 31, 2017

Online Published: February 23, 2017

DOI: $10.5430 /$ crim.v4n2p18

URL: https://doi.org/10.5430/crim.v4n2p18

\begin{abstract}
Pulmonary fibrosis occurs in up to $30 \%$ of patients with pulmonary alveolar proteinosis, and pulmonary hypertension is rarely identified. A previously healthy, highly active, asymptomatic 59-year-old male was diagnosed with autoimmune pulmonary alveolar proteinosis. He was followed clinically for five years until he developed disease progression with dyspnea on exertion. In the interim, he had developed pulmonary fibrosis, and was later found to have pulmonary hypertension. He continued to decline despite aggressive treatment with whole-lung lavage, inhaled granulocyte-macrophage colony-stimulating factor, and rituximab and now has very limited activity, despite use of supplemental oxygen. Pulmonary fibrosis and pulmonary hypertension are uncommon and unpredictable complications of pulmonary alveolar proteinosis. Current treatment regimens for pulmonary alveolar proteinosis may not benefit patients once fibrosis is established. It is not known whether early aggressive treatment can stabilize or delay disease progression.
\end{abstract}

Key Words: Inhaled granulocyte-macrophage colony-stimulating factor, Pulmonary alveolar proteinosis, Pulmonary fibrosis, Pulmonary hypertension, Rituximab

\section{INTRODUCTION}

Pulmonary alveolar proteinosis (PAP) is a rare pulmonary disorder characterized by the accumulation of lipoproteinaceous surfactant in alveoli due to impaired macrophage function. ${ }^{[1]}$ PAP is known for its unpredictable disease course. Up to $31 \%$ of patients with PAP are asymptomatic at presentation, ${ }^{[1,2]}$ and the clinical course is highly variable. ${ }^{[3]}$ Most published therapeutic studies include symptomatic patients only, there- fore little is known of the natural history of PAP in those who are asymptomatic at presentation.

Pulmonary fibrosis is infrequently described in PAP. A review of radiological features of PAP indicates that $30 \%$ of patients have evidence of fibrosis on chest computed tomography (CT), but only 2 of the 27 patients had clinically advanced disease. ${ }^{[4]}$ Although serum biomarkers have been associated with disease severity, these are not routinely used Canada. 
in clinical practice, and there are no validated models to predict who with PAP will deteriorate or develop complications. Outcomes of patients with PAP and fibrotic lung disease are not known, although severity of symptoms has been inversely correlated with diffusing capacity for carbon monoxide (DLCO). ${ }^{[3]}$ Venkateshiah et al. report that non-responders to subcutaneous granulocyte-macrophage colony-stimulating factor (GM-CSF) had worse oxygenation, lower DLCO, lower total lung capacities, and shorter field walking tests. ${ }^{[5]}$ There are no reports on the efficacy of PAP treatments when the disease is complicated by pulmonary fibrosis.

Pulmonary hypertension is also uncommonly reported in PAP, with only four patients identified by $1970 .{ }^{[6]}$ Since that time, little data is available on the prevalence of pulmonary hypertension in PAP. Currently, the prognostic and management implications of pulmonary hypertension in PAP are unknown.

\section{Case presentation}

A healthy 59-year-old male ex-smoker presented with a six month history of persistent cough. His medical history included urinary tract infections and medullary nephrocalcinosis. He had a 20 pack year smoking history. Prior occupations included work in the military and as a marketing consultant. His family history included lung cancer, asbestosis and suspected mesothelioma. Lung function testing revealed an isolated reduction in DLCO (see Table 1). Chest CT had patchy ground-glass opacification, interlobular septal thickening, and intralobular reticulation consistent with "crazy paving" (see Figure 1). Bronchoalveolar lavage and transbronchial biopsies confirmed PAP (see Figure 2). He had an indolent clinical course initially, but five years later developed progressive dyspnea. He was treated with $\mathrm{N}$-acetylcysteine and modified bronchoscopic lobar lavage prior to referral to a subspecialty clinic. A repeat chest CT (see Figure 1) revealed new fibrotic changes, and repeat bronchoscopy confirmed PAP. He now had restrictive lung disease, a reduced DLCO, and a reduced six minute walk distance (see Table 1). Anti GM-CSF antibody testing confirmed autoimmune PAP and no secondary cause was identified.
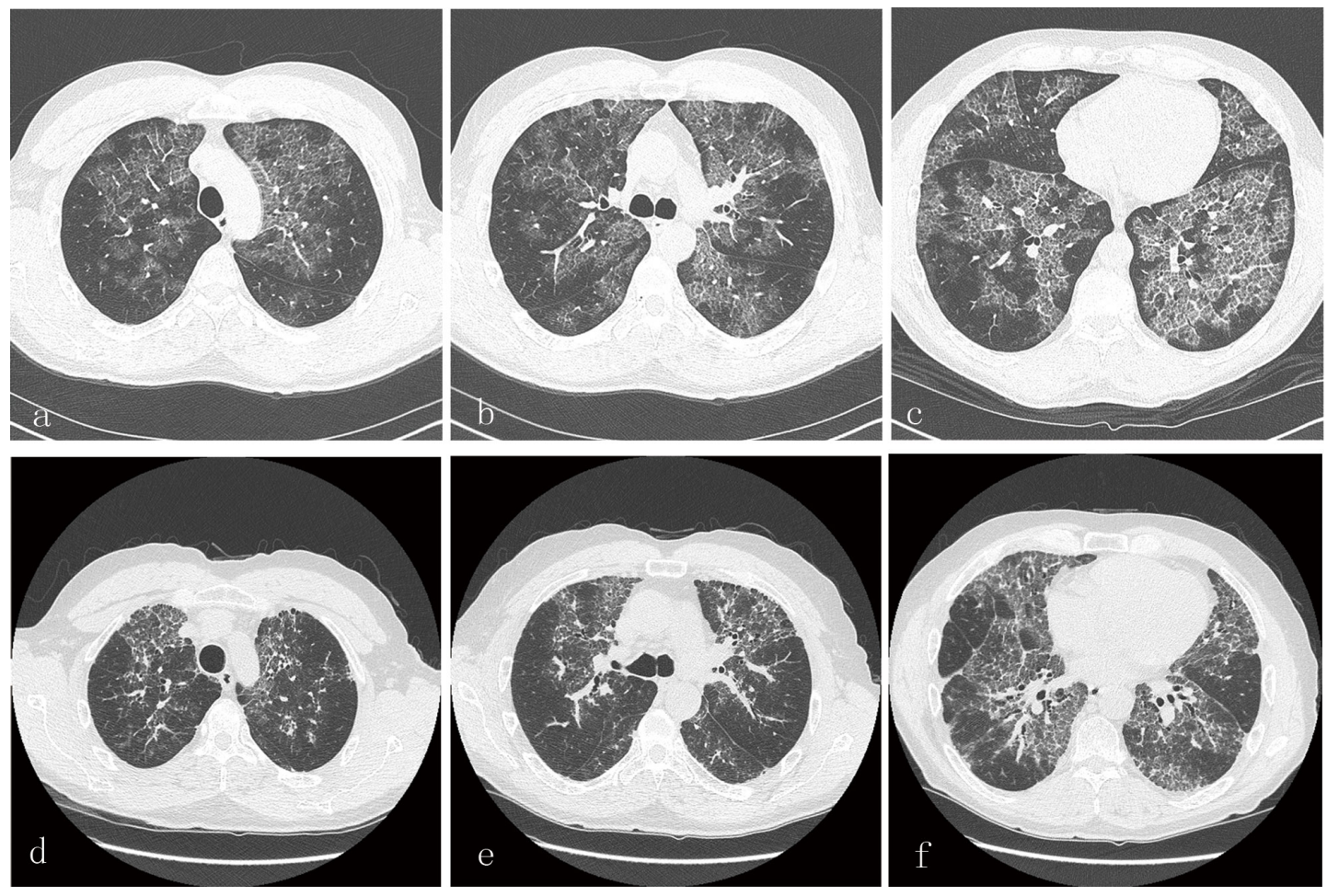

Figure 1. High-resolution computed tomography scans of the chest from 2006 and 2012 reveal pulmonary alveolar proteinosis with fibrosis. High resolution chest computed tomography at the time of initial presentation (2006; a-c) and prior to institution of medical therapy (2012; d-f) in three views (at the level of the [c, f] superior to the diaphragm, (b, e) carina, and [a, d] aortic arch). Images from 2006 reveal patchy geographic changes, interlobular thickening, with significant areas of ground-glass opacification with subpleural sparing, creating polygons classically creating a pattern of "crazy paving". Images from 2012 demonstrate similar findings, associated with some architectural distortion, and mild traction bronchiectasis. 

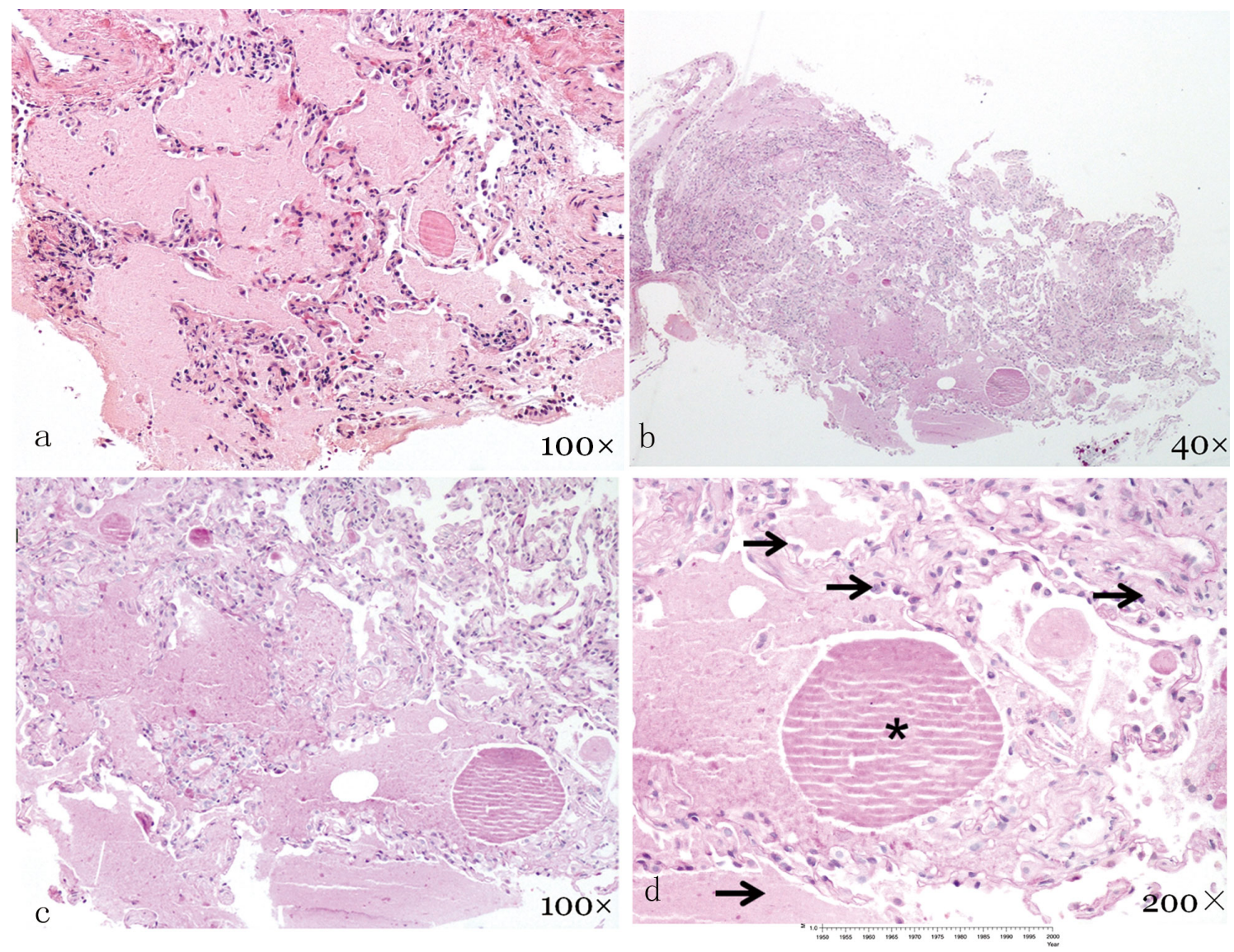

Figure 2. Transbronchial biopsies from 2006. transbronchial biopsies from 2006 confirm the diagnosis of pulmonary alveolar proteinosis. (a) H\&E \& (b-d) periodic acid-Schiff-diastase (PASD) stains, demonstrate granular, acellular lipoproteinaceous material which stains pink with PASD. Inflammatory cells are infrequent. (d) High power magnification reveals hyperplastic type II alveolar cells (arrows). *highlights denser PASD-positive material amongst the more finely granular material. "Cracks" in the PASD material are characteristic.

Table 1. Serial lung function

\begin{tabular}{llllll}
\hline Timing of assessment (month/year) & $\begin{array}{l}\text { FEV1, litres } \\
\text { (\% predicted) }\end{array}$ & $\begin{array}{l}\text { FVC, litres } \\
\text { (\% predicted) }\end{array}$ & $\begin{array}{l}\text { TLC, litres } \\
\text { (\% predicted) }\end{array}$ & $\begin{array}{l}\text { DLCO } \\
\text { (\% predicted) }\end{array}$ & $\begin{array}{l}\text { 6MWD, metres } \\
\text { (nadir oxygen saturation) }\end{array}$ \\
\hline Initial assessment (06/2006) & $3.66(95 \%)$ & $4.74(98 \%)$ & $6.26(85 \%)$ & $18.2(74 \%)$ & $\mathrm{n} / \mathrm{a}$ \\
Prior to treatment initiation (09/2012) & $2.97(81 \%)$ & $3.82(82 \%)$ & $5.23(71 \%)$ & $63 \%$ & $464 \mathrm{~m}(90 \%)$ \\
After first whole lung lavage (10/2013) & $2.67(73 \%)$ & $3.35(73 \%)$ & $\mathrm{n} / \mathrm{a}$ & $58 \%$ & supplemental oxygen initiated \\
After inhaled GM-CSF (04/2014) & $2.60(72 \%)$ & $3.27(71 \%)$ & $4.45(61 \%)$ & $48 \%$ & $363 \mathrm{~m}(80 \%)$ \\
After rituximab (04/2015) & $2.38(66 \%)$ & $3.09(68 \%)$ & $4.23(58 \%)$ & $\mathrm{n} / \mathrm{a}$ & $\mathrm{n} / \mathrm{a}$ \\
After second whole-lung lavage (08/2015) & $2.40(67 \%)$ & $3.07(68 \%)$ & $4.28(58 \%)$ & $45 \%$ & $125 \mathrm{~m}(76 \%)$ \\
\hline
\end{tabular}

Note. FEV1: forced expired volume in one second; FVC: forced vital capacity; TLC: total lung capacity; DLCO: diffusing capacity of the lung for carbon monoxide; 6MWD: six minute walk distance; GM-CSF granulocyte macrophage colony-stimulating factor; n/a: not applicable.

He underwent bilateral whole-lung lavage, inhaled GMCSF (125 mcg inhaled twice daily, alternating weeks for 24 weeks), two series of rituximab infusions (1,000 mg IV every two weeks for two doses per series), followed by a second whole lung lavage. He clinically deteriorated despite this aggressive treatment with progressive lung restriction, decline in DLCO, and functional limitation (see Table 1). Transthoracic echocardiogram demonstrated severe pulmonary hy- pertension (right ventricular systolic pressure $90-95 \mathrm{mmHg}$, right ventricular hypertrophy and a partially flattened intraventricular septum) with normal left ventricular function. He was assessed for lung transplantation, but elected not to proceed. He underwent pulmonary rehabilitation to optimize his functional capacity. 


\section{DISCUSSION}

Despite early diagnosis and subspecialist care, aggressive treatment with serial whole-lung lavage, inhaled GM-CSF and rituximab failed to halt progressive respiratory impairment, pulmonary fibrosis and the subsequent development of pulmonary hypertension in this patient with PAP. The observation that the majority of asymptomatic patients have disease regression or stability without treatment favours an initially conservative approach, acknowledging that evidence is limited. It is not known whether early, aggressive treatment prevents the development of these infrequent complications of PAP. Early reports suggest that younger patients respond less favourably to whole lung lavage; ${ }^{[1]}$ this was not the case with our patient. We do not have clinical prediction models that predict who develops progressive disease. It is important to consider the possibility of negative outcomes or side effects of early, aggressive treatment in minimally symptomatic individuals, as this may outweigh any benefit. A further understanding of the broader clinical experience of response to therapeutics in PAP patients with radiographic or histologic evidence of pulmonary fibrosis may allow clinicians to take different treatment approaches, such as evaluation of the benefit of antifibrotic therapy, if pulmonary fibrosis or pulmonary hypertension limits clinical response to conventional therapies for autoimmune PAP. Lung transplantation may be an option for patients with respiratory failure due to PAP with pulmonary fibrosis; however, PAP has been reported to recur in the transplant lung. ${ }^{[7]}$

\section{ACKNOWLedgements}

The authors would like to thank the patient for allowing us to share and discuss his case.

\section{CONFLicts OF INTEREST Disclosure}

The authors have no competing interests to declare.

\section{REFERENCES}

[1] Seymour JF, Presneill JJ. Pulmonary alveolar proteinosis: Progress in the first 44 years. Am J Respir Crit Care Med. 2002; 166: 216-35. https://doi.org/10.1164/rccm.2109105

[2] Ben-Dov I, Segel MJ. Autoimmune pulmonary alveolar proteinosis: clinical course and diagnostic criteria. Autoimmun. Rev. 2014; 13: 513-517. https://doi.org/10.1016/j. autrev. 2014.01.046

[3] Inoue Y, Trapnell BC, Tazawa R, et al. Characteristics of a large cohort of patients with autoimmune pulmonary alveolar proteinosis in Japan. Am. J. Respir. Crit. Care Med. 2008; 177: 752-62. https://doi.org/10.1164/rccm. 200708-12710C

[4] Venkateshiah SB, Yan TD, Bonfield TL, et al. An open-label trial of granulocyte macrophage colony stimulating factor therapy for moderate symptomatic pulmonary alveolar proteinosis. Chest. 2006; 130: 227-37. https ://doi.org/10.1378/chest.130.1.227

[5] Holbert JM, Costello P, Li W, et al. CT features of pulmonary alveolar proteinosis. Am. J. Roentgenol. 2001; 176: 1287-94. https: //doi.org/10.2214/ajr.176.5.1761287

[6] Olivia R, Vogel JHK. Reactive pulmonary hypertension in alveolar proteinosis. Chest. 1970; 58: 167-9. https://doi.org/10.1378/ chest.58.2.167

[7] Parker LA, Novotny DB. Recurrent alveolar proteinosis following double lung transplantation. Chest. 1997; 111(5): 1457-58. https://doi.org/10.1378/chest.111.5.1457 